Gilbert Lupfer

\title{
Bibliotheken und Kunstsammlungen in Dresden. Eine Familiengeschichte
}

\section{Unter einem Fürstendach im Residenzschloss}

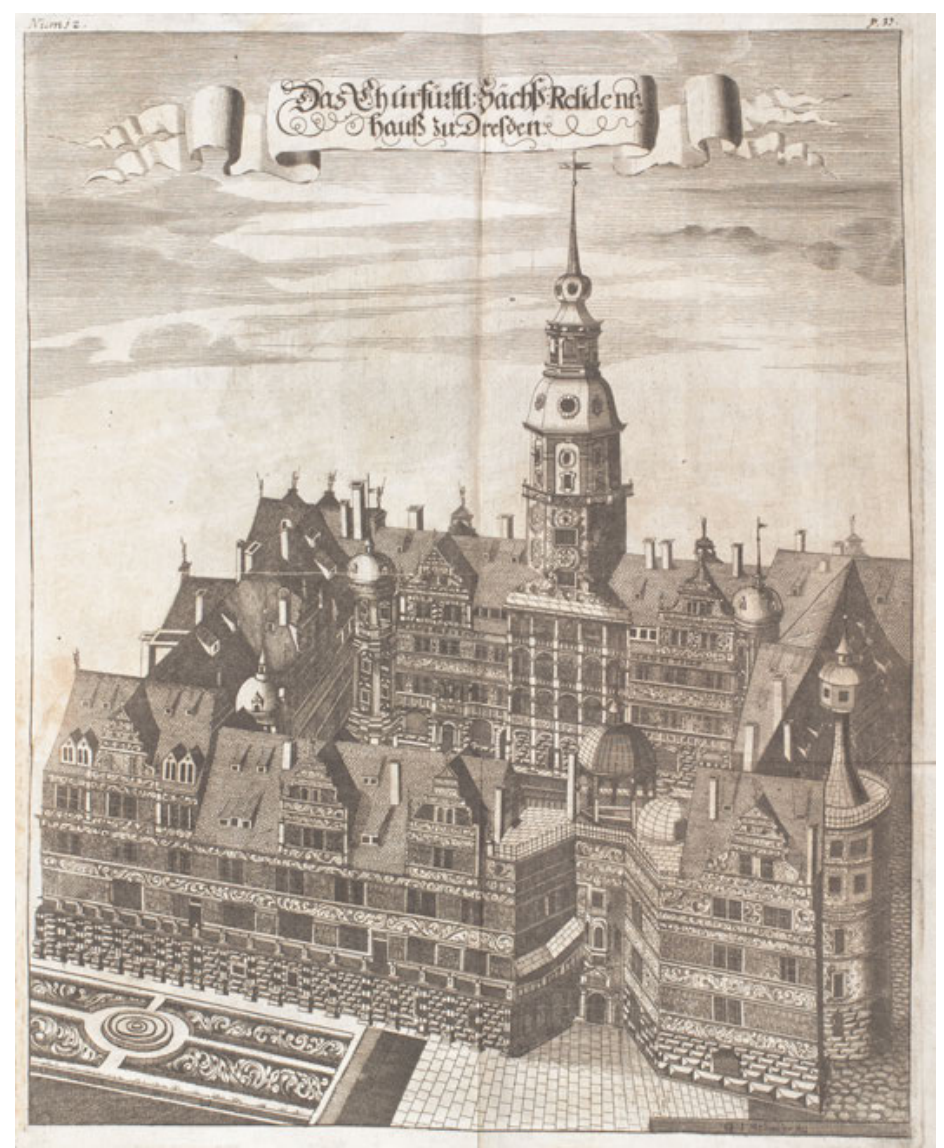

Abb. 1: G. I. Schneider: Das Residenzschloss Dresden. Abgedruckt in: Anton Weck: Der ChurFürstlichen Sächsischen weitberuffenen Residentz- und Haupt-Vestung Dresden Beschreibung und Vorstellung, 1680. SLUB Dresden/Deutsche Fotothek.

„Zukunft seit 1560“ verkündeten die Staatlichen Kunstsammlungen Dresden 2010 als Motto ihres 450. Geburtstages, der auf das Gründungsdatum der Kunst- 
kammer im kurfürstlichen Residenzschloss zurückgeht - präziser gesagt auf ein 100 Jahre später vom Kunstkämmerer Tobias Beutel d. Ä. festgelegtes, möglicherweise fiktives Gründungsdatum. ${ }^{1}$,Wir haben aber schon 2006 unseren 450. Geburtstag gefeiert“, durfte da die Sächsische Landesbibliothek stolz entgegensetzen, denn der erste Hinweis auf eine systematische Büchersammlung unter demselben Dach des Dresdner Residenzschlosses soll auf 1556 datieren. $^{2}$ „Glückwunsch, gewonnen!“ - könnte man vonseiten der Kunstsammlungen antworten, und gleichzeitig doch wieder leise triumphieren, denn bereits 1539 wurde im Schloss eine Harnischkammer eingerichtet, aus der später die Rüstkammer hervorging und von 1543 stammt der erste Hinweis auf eine kurfürstliche Silbersammlung (ob es sich dabei um museale Sammlungen in einem späteren Sinne handelte, sei dahingestellt).

Lassen wir offen, wer oder was nun wirklich älter ist. Jedenfalls drängt es sich auf, zu einer Familienmetapher zu greifen und Bibliothek sowie Kunstsammlungen als Geschwister anzusprechen, als meist friedlich zusammenlebende und sich gegenseitig bereichernde, manchmal aber auch als rivalisierende oder als einander etwas fremde Geschwister - wie das eben in Familien der Fall ist. Verfolgen wir nun einige wichtige Stationen in der Entwicklung der Dresdner Sammlungen vom 16. Jahrhundert bis heute.

Das gemeinsame Aufwachsen der Sammlungen unter einem Dach Mitte des 16. Jahrhunderts währte nicht lange und wurde bald unterbrochen, denn 1574 , die Bibliothek war noch nicht einmal 20 Jahre alt, musste sie ins gerade fertiggestellte Jagdschloss Annaburg bei Torgau umziehen. Ein erster Katalog verzeichnete in diesem Jahr 1721 Positionen. Als die namengebende Kurfürstenwitwe Anna verstorben war, holte ihr Sohn Christian I. die Bibliothek 1586 nach Dresden zurück; ihre Aufstellung fand sie im Residenzschloss direkt neben der schwesterlichen Kunstkammer. Deren erstes Inventar von 1587 verzeichnete rund 10000 Stücke aller Art, von der Smaragdstufe aus Kolumbien über die Tischsonnenuhr aus Augsburg und den Schneckenbohrer aus Nürnberg bis zu

1 Vgl. zum Folgenden vor allem Kolb, Karin, Gilbert Lupfer u. Martin Roth (Hrsg.): Zukunft seit 1560. Die Chronik. Bearbeitet von Volkmar Billig. Berlin, München: Deutscher Kunstverlag 2010; auf Einzelverweise bei den Ereignissen wird im Folgenden weitgehend verzichtet. Für ihre Unterstützung danke ich besonders Frau Dr. Maria Obenaus und Herrn Dr. Thomas Rudert.

2 Vgl. zur Bibliotheksgeschichte Frühauf, Wolfgang: Von der kurfürstlichen Privatbibliothek zur Sächsischen Landesbibliothek. In: Von der Liberey zur Bibliothek. 440 Jahre Sächsische Landesbibliothek. Hrsg. von Günter Gattermann. Dresden: Sandstein 1996. S. 13-27; Bürger, Thomas: Wandel und Kontinuität in 450 Jahren. Von der kurfürstlichen Liberey zur Sächsischen Landesbibliothek - Staats- und Universitätsbibliothek Dresden. In: Wissenschaftliche Zeitschrift der TU Dresden 55 (2006) H. 1-2. S. 29-36. 
Giambolognas bronzenem Merkur aus Florenz. ${ }^{3}$ Um 1600 blühten und wuchsen die Sammlungen unter Christian I. und seinem Sohn Christian II. Das Kunstkammerinventar von 1619 dokumentiert eine beachtliche Erweiterung: So wurden 1590 Elfenbeinlöffel aus Westafrika erworben, wohl die ersten Ethnographica in der Kunstkammer. Einen gewichtigen Zuwachs erfuhr beispielsweise auch die Münzabteilung der Kunstkammer, etwa durch 1299 in Augsburg gekaufte römische und byzantinische Münzen. Nicht schlechter erging es der Bibliothek: Zwei Büchersammlungen, vom Chemnitzer Gelehrten Georg Fabricius und vom Stolberger Kanzler Dietrich von Werthern, verdoppelten den Bestand auf nunmehr 5688 Bücher (im Jahr 1595). ${ }^{4}$ Platzprobleme zeichneten sich hier wie dort ab.

\section{Emanzipation im Zwinger}

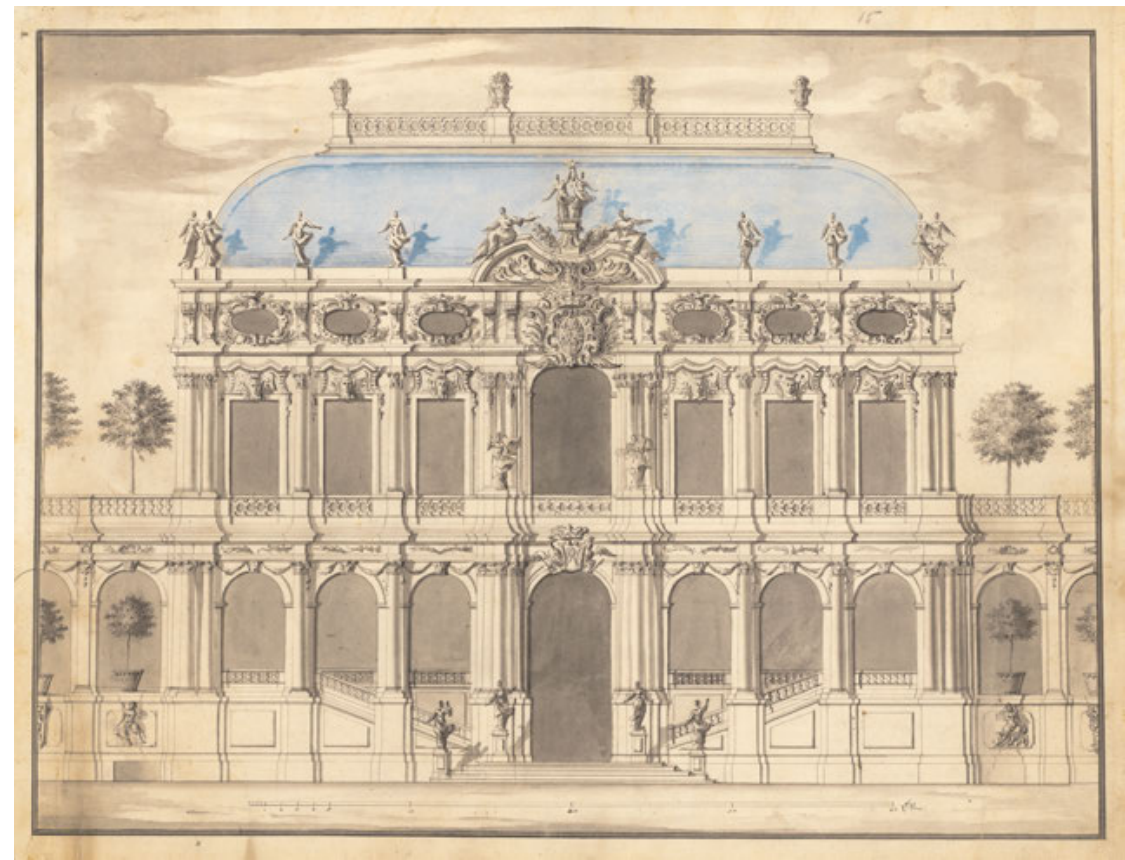

Abb. 2: Matthäus Daniel Pöppelmann: Entwurf zu einem Zwingerpavillon, um 1715. SLUB Dresden/Deutsche Fotothek.

$3 \mathrm{Zu}$ den Inventaren der kurfürstlich-sächsischen Kunstkammer in Dresden von 1587, 1619, 1640 und 1741 vgl. Syndram, Dirk u. Martina Minning (Hrsg.): Die kurfürstlich-sächsische Kunstkammer in Dresden. 4 Bde. Dresden: Sandstein 2010.

4 Vgl. Frühauf, Von der kurfürstlichen Privatbibliothek (wie Anm. 2), S. 16. 
Überspringen wir - auch aus Platzgründen - das 17. Jahrhundert und kommen zum für jede Art von Sammlungen goldenen Augusteischen Zeitalter. Erwerbungen fast ohne Zahl, die Ausdifferenzierung der Bestände und die Verwissenschaftlichung des Sammlungsmanagements mögen Schlagworte für die Entwicklung sein. August der Starke beauftragte 1713 seinen Leibarzt, den Botaniker Johann Heinrich Heucher, ${ }^{5}$ mit der wissenschaftlichen Neuordnung der Bestände der Kunstkammer. Es war der Anfang vom Ende dieser Universalsammlung, die zugunsten der sich herausbildenden Spezialkollektionen ,geplündert" wurde. Auch der Bestand der Bibliothek profilierte sich, denn sie erhielt einerseits Bücher aus der Kunstkammer und gab andererseits Kunstwerke ab. ${ }^{6}$ Das war allerdings keine Dresdner Besonderheit, denn in ganz Europa endete die hohe Zeit der universalen Kunst- und Wunderkammern. Ohne ihren ganz eigenständigen Charakter zu leugnen, kann man auch die Bibliothek als eine dieser zunehmend wissenschaftlich ausgerichteten Spezialsammlungen bezeichnen. 1720 zogen eben diese wissenschaftlichen Sammlungen, nämlich das Kupferstich-Kabinett, das Münzkabinett, das Antiken-Kabinett, das Naturalienkabinett und die Anatomiekammer zusammen mit der Bibliothek aus dem Schloss ins neue „Regimentshaus“ am Jüdenhof (Neumarkt). Ernst Christoph Graf von Manteuffel wurde zum „Directeur-General unserer Bibliothequen und Cabinette“ ernannt, der bereits erwähnte Leibarzt Heucher zum „General- und Spezial-Inspector“. Natürlich war mit diesem Schritt hinaus aus der unmittelbaren Umgebung des Fürsten in der Residenz (sofern er sich denn dort aufhielt) auch eine gewisse Öffnung für eine adlige und gelehrte Öffentlichkeit verbunden, von einem allgemeinen Zugang konnte jedoch noch keine Rede sein.

Die Bibliothek wuchs, etliche große adlige Privatbestände konnten erworben werden und so blieb die neue räumliche Lösung nur von kurzer Dauer, zumal auch die meisten anderen Sammlungen auf rapiden Zuwachs angelegt waren. Außer dem Münzkabinett zogen nicht einmal ein Jahrzehnt später, 1728, die wissenschaftlichen Sammlungen, zu denen die Bibliothek gehörte, in den nun zum „Palais des Sciences“ promovierten Zwinger; die Bibliothek konnte sich dort in immerhin drei Pavillons ausbreiten. ${ }^{7}$ Auch die inzwischen reichlich dezimierte Kunstkammer landete übrigens nach mehreren Zwischenstationen 1733 wieder im Zwinger. 1738 übernahm Graf Heinrich von Brühl die Oberauf-

5 Biografische Angaben zu den im Aufsatz genannten Personen vgl. Zukunft seit 1560 (wie Anm. 1) sowie die Sächsische Biografie - das personengeschichtliche Lexikon zur Geschichte Sachsens unter http://saebi.isgv.de.

6 Vgl. Aurich, Frank u. Nadine Kulbe: Geordnetes Wissen. Die Bücher in der Kunstkammer am Dresdner Hof. In: Die kurfürstlich-sächsische Kunstkammer in Dresden. Geschichte einer Sammlung. Hrsg. von Dirk Syndram u. Martina Minning. Dresden: Sandstein 2012. S. 293-329. 7 Vgl. Frühauf, Von der kurfürstlichen Privatbibliothek (wie Anm. 2), S. 17. 
sicht über alle Sammlungen und war damit beteiligt an einer für Sachsen fiskalisch mittelfristig fatalen, langfristig jedoch höchst segensreichen kulturellen Expansion. ${ }^{8}$

1739 erwarb die Bibliothek über einen Vermittler in Wien ihr bis heute herausragendes Stück, sozusagen ihre „Mona Lisa“, nämlich den Codex Dresdensis, besser bekannt als Maya-Codex. Es sollte 15 Jahre dauern, bis auch die Kunstsammlungen endlich zu ihrer „Mona Lisa“ gelangten, zu Raffaels Sixtinischer Madonna, die den Mönchen von San Sisto in Piacenza teuer abgekauft wurde. ${ }^{9}$ Die Sixtina war die Krönung einer gigantischen Einkaufskampagne, die das Profil der Dresdner Sammlungen, vor allem der Gemäldegalerie und der Skulpturensammlung, bis heute prägt. Man kann an dieser zeitlichen Stelle kurz eine wichtige Personalie einschieben, um vielfältige personelle, strukturelle und inhaltliche Verflechtungen zu charakterisieren: Carl Heinrich von Heineken, bis dato Privatsekretär und Privatbibliothekar des in Sachsen zunehmend tonangebenden Grafen von Brühl, wurde 1746 zum Inspektor des Kupferstich-Kabinetts ernannt, um dessen Ausbau und Profilierung er sich bleibende Verdienste erwarb.

Zum Ende des Siebenjährigen Krieges 1763 erfolgte ein Einschnitt in der sächsischen Geschichte; das Jahr steht für einen politischen und ökonomischen Absturz und den Beginn entbehrungsreicher Jahre in Sachsen. Immerhin konnte die Bibliothek, im Unterschied zu den Kunstsammlungen, quantitativ wie qualitativ nennenswert wachsen. 1765 wurde die mehr als 40000 Bände umfassende Bibliothek des 1762 verstorbenen Gelehrten und Staatsmannes Heinrich von Bünau erworben, wohl eine der wichtigsten privaten Büchersammlungen des 18. Jahrhunderts in Deutschland; berühmt wurde sie nicht zuletzt durch ihren zeitweiligen Bibliothekar Johann Joachim Winckelmann. ${ }^{10}$ Heinrich von Bünau hatte in scharfer persönlicher wie politischer Konkurrenz zum Kanzler Heinrich Graf von Brühl gestanden, letztendlich unterlag er. Diese Konkurrenz hatte sich auch auf das Sammeln von Büchern erstreckt. So mutet es wie eine Ironie der Geschichte an, dass 1768 auch die mehr als 60000 Bände umfassende Bibliothek des 1763 in Schimpf und Schande verstorbenen Brühl in der kurfürstlichen Sammlung aufging. Die nicht weniger bedeutenden Gemälde- und Grafikkollektionen des ehemaligen Kanzlers konnte Sachsen dagegen nicht im Lande halten (schließlich gab es noch kein Gesetz zum Schutz national wertvol-

8 Vgl. Koch, Ute C. u. Cristina Ruggero (Hrsg.): Heinrich Graf von Brühl. Ein sächsischer Mäzen in Europa. Akten der internationalen Tagung zum 250. Todesjahr. Dresden: Sandstein 2017. 9 Vgl. Brink, Claudia u. Andreas Henning (Hrsg.): Raffael. Die Sixtinische Madonna. Geschichte und Mythos eines Meisterwerks. München, Berlin: Deutscher Kunstverlag 2005.

10 Zur „Bibliotheca Bünaviana“ vgl. Nitzschke, Katrin: Die großen Erwerbungen des 18. Jahrhunderts. In: Von der Liberey zur Bibliothek (wie Anm. 2). S. 29-41, hier S. 35-36. 
len Kulturguts): Sie gingen nach St. Petersburg und bildeten dort einen Grundstock der Eremitage.

\section{Öffentlichkeit im Japanischen Palais}

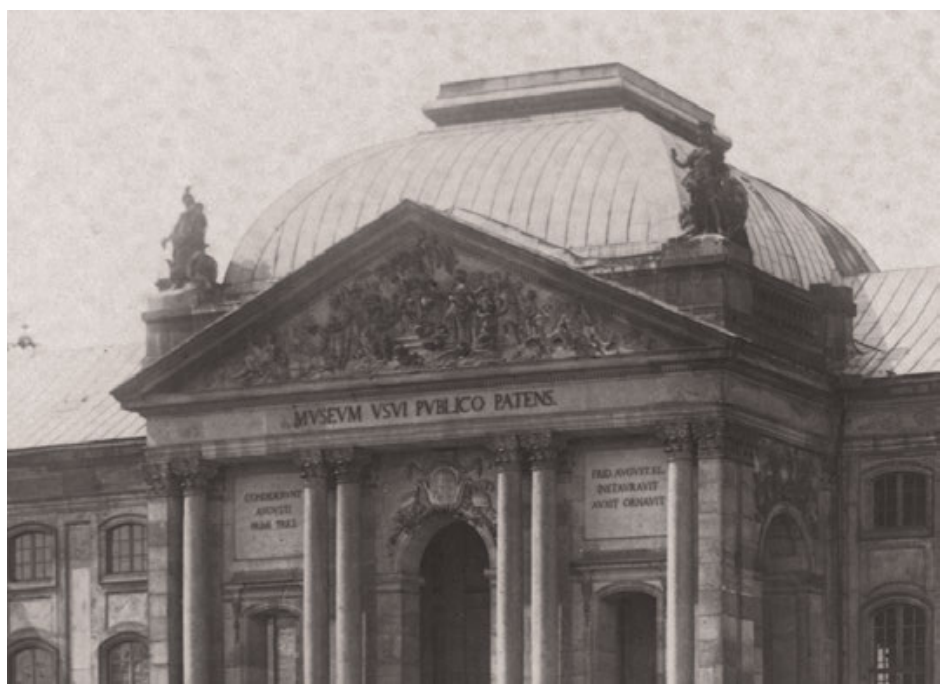

Abb. 3: Japanisches Palais mit Giebelinschrift „MUSEO USUI PUBLICO PATENS“ (Museum zum öffentlichen Gebrauch), Foto: Hermann Krone (Detail), 2. Hälfte 19. Jahrhundert. SLUB Dresden/Deutsche Fotothek.

Die letzten anderthalb Jahrzehnte des 18. Jahrhunderts markieren einen Meilenstein nicht nur in der sächsischen, sondern überhaupt in der europäischen Museums- und Sammlungsgeschichte, nämlich den sanften und sukzessiven Übergang von der Exklusivität der herrschaftlichen Privatsammlung zur öffentlichen Sammlung modernen Charakters. Kurfürst Friedrich August III. hatte „den Entschluss gefasst, Unsere Bibliothek nebst den Anticken- und MünzSammlungen in das sogenannte Holländische Palais verlegen zu lassen“. ${ }^{11}$ Dieser Umzug wurde unter anderem durch den Verkauf von Buch-Dubletten finanziert. Das heute als Japanisches Palais bekannte Gebäude erhielt 1788 die Giebelinschrift „MUSEO USUI PUBLICO PATENS“, es wurde also einer tendenziell

11 Königliches Rescript vom 18. September 1784. Zit. nach Boller, Hildegard Gabriele: Die Dresdner Antikensammlung. In: Tempel der Kunst. Die Geburt des öffentlichen Museums in Deutschland 1701-1815. Hrsg. von Bénédicte Savoy. Mainz: von Zabern 2006. S. 117-144, hier S. 128. 
schon bürgerlichen Öffentlichkeit gewidmet (wobei man sich hinsichtlich des Adressatenkreises keine Illusionen machen sollte: Der Bergmann aus Freiberg gehörte wohl genauso wenig zum potenziellen Besucherkreis wie die Weingärtnerin aus Meißen). In diesem also fast schon demokratisch gedachten $\mathrm{Mu}$ seumsgebäude, das August der Starke noch ein halbes Jahrhundert zuvor als sein exklusives Porzellanschloss angesehen hatte, waren die Antikensammlung, das Münzkabinett und die Bibliothek untergebracht. Letztere erhielt den Namen „Königliche Öffentliche Bibliothek“ und mit Johann Christoph Adelung einen der bedeutendsten Bibliothekare seiner Zeit. 1807 übrigens stattete Napoleon höchstpersönlich dem Japanischen Palais einen Besuch ab; da Sachsen ein Verbündeter Frankreichs war, blieb es zum Glück beim begehrlichen Blick. ${ }^{12}$

Das Japanische Palais avancierte zu einem Motor der kulturellen Entwicklung in Sachsen: 1815 wurde hier der freie Eintritt, zunächst nur an einigen Wochentagen, erprobt, später auch in anderen Museumsgebäuden. Die frühen 1830er Jahre bedeuteten dann in zweierlei Hinsicht eine nächste Zäsur. 1831 wurde im Rahmen einer - mit dem Namen Bernhard von Lindenau verbundenen - sächsischen Verfassungsreform auch der Status der Sammlungen verändert. Sie blieben zwar im Eigentum des Königs, aber nun als unveräußerliches Hausfideikommiss, das heißt, sie waren der willkürlichen Verfügung des Herrschers entzogen. 1832 kamen mehr als zweieinhalb Jahrhunderte Museumsgeschichte zu einem traurigen Ende, denn die als kläglicher Rest einstiger Pracht und Fülle zuletzt im Zwinger beheimatete Kunstkammer wurde endgültig aufgelöst, die Restbestände auf die Sammlungen verteilt oder verkauft.

Die Bibliothek entwickelte sich im Laufe des 19. Jahrhunderts zur gewichtigsten Sammlung im Japanischen Palais, der nach und nach die anderen weichen mussten: die Porzellansammlung aus dem Souterrain 1876 ins Johanneum am Neumarkt, die Antikensammlung in das in den 1890er Jahren zum Museumsgebäude umgebaute und Albertinum genannte ehemalige Zeughaus. Eine interessante Personalunion sei in diesem Zusammenhang erwähnt: Der aus Chemnitz stammende Gustav Friedrich Klemm, Sekretär der Bibliothek seit 1831 (und ab 1852 ihr Leiter) wurde ab 1834 auch Leiter der Porzellansammlung.

Die Mitte des 19. Jahrhunderts brachte den Siegeszug eines neuen Mediums, wohl kaum weniger gesellschaftsverändernd als die Siegeszüge diverser neuer Medien im 20. Jahrhundert - den der Fotografie. Die Dresdner Museen erkannten früh die Zeichen der Zeit. 1850 fertigte der Dresdner Fotopionier Hermann Krone kurz vor dem Umzug ins neue Galeriegebäude, gerade noch am alten Galeriestandort im Johanneum, die erste Daguerrotypie der Sixtina an und

12 Vgl. Boller, Dresdner Antikensammlung (wie Anm. 11), S. 127; Frühauf, Von der kurfürstlichen Privatbibliothek (wie Anm. 2), S. 21. 
revolutionierte damit mittelfristig die Möglichkeiten der Reproduktion von $\mathrm{Mu}$ seumsexponaten. Das Kupferstich-Kabinett begann dann in den 1890er Jahren unter der Leitung von Max Lehrs als erstes Museum in Deutschland, systematisch „Künstlerische Fotografie“ zu sammeln - wie überhaupt einige der Dresdner Museen und ihre Wissenschaftler zum Jahrhundertende ausgesprochen innovativ und trendsetzend waren.

1870 wurden die Königlichen Sammlungen für Kunst und Wissenschaft, zu denen neben rund einem Dutzend Museen nach wie vor auch die Königliche Öffentliche Bibliothek gehörte, neu strukturiert. Sie unterstanden nun einer Generaldirektion mit einem Minister (zunächst war es der Kultus-, später der Finanzminister) als Vorstand. Das operative Geschäft führte der Vortragende Rat in der Generaldirektion. Ab 1885 war das Woldemar von Seidlitz, eine für das Dresdner Kulturleben an der Wende vom 19. zum 20. Jahrhundert enorm wichtige, prägende Persönlichkeit. Seidlitz ging 1919 in den Ruhestand.

\section{Die Familienbande lösen sich}

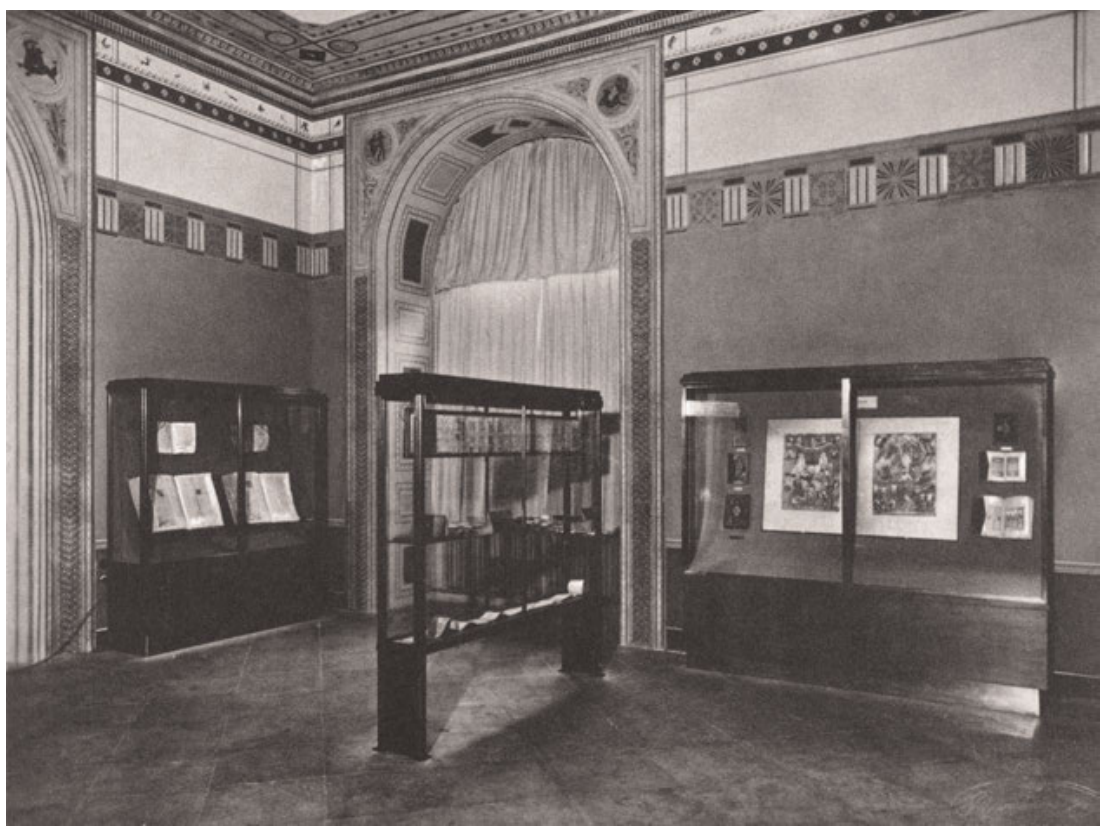

Abb. 4: Sächsische Landesbibliothek im Japanischen Palais. Handschriftensaal im Buchmuseum, um 1935. Foto: Wilhelm Moeck. SLUB Dresden/Deutsche Fotothek. 
Nun standen wirklich große, einschneidende Veränderungen an. Aus den Königlichen Sammlungen wurden die Staatlichen Sammlungen, die ministerielle Zuständigkeit ging an das Ministerium des Kultus und öffentlichen Unterrichts über. Die Sächsische Landesbibliothek blieb immer noch im großen Sammlungsverbund, doch faktisch wurde die Distanz zwischen der inzwischen alleine im Japanischen Palais residierenden Bibliothek und den Museen wohl größer. Eine komplizierte Angelegenheit verband die meisten Sammlungen: der so genannte Auseinandersetzungsvertrag des Freistaats Sachsen mit dem Haus Wettin, der den Wettinern als Entschädigung für den mit dem Ende der Monarchie 1918 verlorenen Thron neben Immobilien und Ländereien auch eine Vielzahl an Kulturgütern zugestand; die Sammlungsleiter mussten lange Listen auszusondernder und an das Haus Wettin zu übergebender Objekte anfertigen. ${ }^{13}$ In ihrer Substanz trafen die Verluste die Sammlungen jedoch glücklicherweise nicht. Die „Auseinandersetzung“ mit den Wettinern allerdings war damit noch lange nicht abgeschlossen, auf sie wird zurückzukommen sein.

Die Weimarer Republik war für Museen keine Blütezeit, die Etats für den Unterhalt und die Erweiterung des Bestands waren fast überall sehr klein. Immerhin konnte Galeriedirektor Hans Posse für sein Museum diesen Mangel mit Hilfe eines potenten Freundeskreises, zu dem vor allem jüdische Sammler und Mäzene aus Dresden gehörten, halbwegs ausgleichen. Die schon ausgearbeiteten Pläne für eine Erweiterung des Galeriegebäudes am Zwinger - der erste Spatenstich war noch kurz vor dem Ersten Weltkrieg erfolgt - wurden jedoch aus finanziellen Gründen zur Makulatur. Die Sächsische Landesbibliothek hingegen konnte sich daran erfreuen, dass das Japanische Palais ab 1927 zum funktionalen, modernen Bibliotheksgebäude - ohne andere Nutzer - umgebaut wurde. Mit der Eröffnung eines Buchmuseums 1935 fanden die umfangreichen Umbaumaßnahmen ihren Abschluss.

Doch Freude darüber mag man retrospektiv wenig empfinden, denn das Jahr 1933 hatte alles verändert - und Dresden spielte leider keine positive Rolle in dieser Entwicklung. 1933 fand auf dem Wettiner Platz eine Bücherverbrennung statt - und im Rathaus eine Ausstellung „Entarteter Kunst“. Von wenigen Aktivisten wie Hermann Fichtner (Direktor des Kunstgewerbemuseums und der Porzellansammlung) abgesehen, spielten die Museen und Sammlungen immerhin keine besonders aktive Rolle in der völkischen Ausrichtung. ${ }^{14}$ Das fatale En-

13 Vgl. Rudert, Thomas: Die Staatlichen Sammlungen für Kunst und Wissenschaft Dresden und die Vermögensauseinandersetzung mit dem Haus Wettin 1918-1927. In: Jahrbuch der Staatlichen Kunstsammlungen Dresden 33 (2006-2007). S. 83-113; Rudert, Thomas u. Gilbert Lupfer: Die Dresdner Kunstsammlungen und die Vermögensauseinandersetzung mit dem Haus Wettin 1924. Historische Grundlagen aktueller Eigentumsfragen. In: Museumskunde 73 (2008) H. 1. S. 50-56. 
gagement des Galeriedirektors Posse und seines Nachfolgers Hermann Voss im Rahmen von Adolf Hitlers „Sonderauftrag Linz“ sei hier der Kürze halber außer Acht gelassen und auf die einschlägigen Publikationen verwiesen. ${ }^{15}$ Der von Deutschland ausgegangene Krieg kam 1945 auch nach Dresden, mit verheerenden Folgen. Der Großteil der Sammlungsgebäude vom Zwinger bis zum Japanischen Palais wurde in der Nacht des 13. Februar 1945 schwer beschädigt (lediglich das Albertinum blieb nutzbar). Während aber die rechtzeitig nach rund vier Dutzend Orten außerhalb Dresdens ausgelagerten Bestände der Museen die Luftangriffe halbwegs unbeschadet überstanden, stellte sich die Bilanz für die Landesbibliothek schlechter dar, denn Tausende von Büchern im Japanischen Palais wurden, vor allem durch Löschwasser, zerstört oder gravierend geschädigt. Wenige Monate nach dem Ende des Zweiten Weltkriegs standen, wie viele Institutionen im Osten Deutschlands, auch die Dresdner Sammlungen vor einem schwierigen, prekären Neubeginn, denn ein Großteil ihrer Bestände wurde von den Trophäenbrigaden der Roten Armee in die Sowjetunion abtransportiert und verschwand dort für Jahre fast spurlos. ${ }^{16}$

Die Dresdner Gemäldegalerie erhielt den Großteil ihrer Werke 1955/56 quasi in einem sowjetischen Pilotprojekt - zurück, die anderen Museen auf dem Gebiet der DDR dann 1958, jeweils allerdings mit deutlichen Lücken. Die Landesbibliothek hingegen wartet nach wie vor auf die Rückkehr ihrer Kriegsverluste, die mehr als 200000 Bände umfassen und von denen sich zumindest ein wesentlicher Teil noch in russischen Bibliotheken befindet. ${ }^{17}$ Aber auch der eine oder andere Schatz aus dem Dresdner Museumsbestand wird heute noch von russischen und ukrainischen Museen und vor allem privaten Sammlern „gehütet“.

14 Die Staatlichen Kunstsammlungen Dresden erforschen derzeit in einem von der Deutschen Forschungsgemeinschaft geförderten Projekt die Staatlichen Sammlungen für Kunst und Wissenschaft in Dresden, ihre Direktoren und wissenschaftlichen Mitarbeiter in der Zeit des Nationalsozialismus (Projektlaufzeit 2016-2019).

15 Vgl. Staatliche Kunstsammlungen Dresden, Gilbert Lupfer u. Thomas Rudert (Hrsg.): Kennerschaft zwischen Macht und Moral. Annäherungen an Hans Posse (1879-1942). Köln, Weimar, Wien: Böhlau 2015; Iselt, Kathrin: „Sonderbeauftragter des Führers“. Der Kunsthistoriker und Museumsmann Hermann Voss (1884-1969). Köln, Weimar, Wien: Böhlau 2010.

16 Vgl. dazu die Beiträge von Frank Aurich sowie Gilbert Lupfer in: Kulturgüter im Zweiten Weltkrieg. Verlagerung - Auffindung - Rückführung. Hrsg. von der Koordinierungsstelle für Kulturgutverluste Magdeburg. Bearbeitet von Uwe Hartmann. Magdeburg: Koordinierungsstelle für Kulturgutverluste 2007. S. 131-141 u. 267-285.

17 Vgl. Bürger, Wandel und Kontinuität (wie Anm. 2), S. 35. 
Die frühen 1950er Jahre sahen alle Dresdner Kulturinstitutionen in einer Art Notbetrieb mit bescheidensten Mitteln, in ruinösen Räumen. ${ }^{18}$ Strukturell und organisatorisch fanden tief greifende Veränderungen statt. 1953 wurden die Kunstsammlungen von den wissenschaftlichen Sammlungen sowie von der Landesbibliothek getrennt, der schwesterliche Verbund endgültig aufgelöst ein gravierender Einschnitt nach fast vier Jahrhunderten gemeinsamer Geschichte. Die Kunstsammlungen betrieben nun mit der Kunstbibliothek in der Güntzstraße, in den früheren Räumen von Kunstgewerbeschule und -museum, eine eigene Bücherei mit allerdings zunächst recht lückenhaftem, willkürlich zusammengesetztem Bestand.

Mit der bereits erwähnten großen Rückführungsaktion 1955/58 und der baulichen Wiederherstellung der Museumsgebäude (mit Ausnahme des Residenzschlosses) kehrte für die Kunstsammlungen nach und nach Nachkriegsnormalität ein, sie entwickelten sich bald schon zum - neben Berlin - führenden $\mathrm{Mu}$ seumsverbund der DDR mit großer internationaler Ausstrahlung. Der Neustart für die Landesbibliothek gestaltete sich wesentlich schwieriger: Untergebracht in einer ehemaligen Kaserne am nördlichen Stadtrand, bar eines Großteils ihrer Bestände, folgten durchaus entbehrungsreiche, immer vom Geist des sich verfestigenden Provisoriums gekennzeichnete Jahre und Jahrzehnte; auch nach der Vereinigung mit der genauso unter prekären räumlichen Verhältnissen leidenden Bibliothek der TU Dresden 1996 änderte sich daran zunächst wenig.

Dieser provisorische Status gehörte erst im Jahr 2002 der Vergangenheit an, als der Neubau am Zelleschen Weg auf dem Unicampus eröffnet wurde und die Sächsische Landesbibliothek - Staats- und Universitätsbibliothek (SLUB) es unter dem Generaldirektorat von Thomas Bürger schnell schaffte, sich in der Spitzengruppe der deutschen und europäischen Bibliotheken festzusetzen. Führend ist die SLUB unter anderem auf dem Feld der Digitalisierung, wo sie wiederum auf die Staatlichen Kunstsammlungen trifft, die mit ihrem Digitalisierungsprojekt „Daphne“ eine Schrittmacherrolle in Deutschland spielen. Obwohl es sich um zwei getrennte Institutionen handelt, könnte auf vielen Feldern die Zusammenarbeit zwischen Kunstsammlungen und Bibliothek kaum besser und vertrauensvoller sein, auch im Rahmen einer Forschungspartnerschaft mit anderen Institutionen der Spitzenforschung unter dem Label DRESDEN-concept.

18 Vgl. Rudert, Thomas: Auf Messers Schneide. Vom schwierigen Neuanfang in den Dresdner Staatlichen Sammlungen für Kunst und Wissenschaft nach dem Ende des Zweiten Weltkriegs. In: „So fing man einfach an, ohne viele Worte“. Ausstellungswesen und Sammlungspolitik in den ersten Jahren nach dem zweiten Weltkrieg. Hrsg. von Julia Friedrich u. Andreas Prinzing. Berlin: de Gruyter 2013. S.186-194. 
Eine Pointe allerdings muss am Ende dieser Skizze stehen, denn beide Institutionen wurden um 2008 von ihrer gemeinsamen Vergangenheit eingeholt. Das Haus Wettin reklamierte die Rückgabe von Tausenden von Kunstwerken, anderen Kulturgütern und auch Büchern, die gleich nach dem Kriegsende 1945 von der sowjetischen Besatzungsmacht in den Schlössern Moritzburg und Wachwitz beschlagnahmt und enteignet worden waren und von denen manche dann, bisweilen auf verschlungenen Wegen, in Museen und Bibliotheken gelangten. Die Forderungen wirkten zunächst äußerst bedrohlich, in allen Häusern begannen umfangreiche, systematische Recherchen, die über Jahre den Alltag mitprägten und erst nach der peinlich genauen Überprüfung der Provenienz fast schon unzähliger Objekte (und der Entkräftigung zahlreicher Positionen auf den Forderungslisten) zu einem glücklichen Ende kamen. Am 17. Juli 2014 wurde in den Räumen der SLUB ein abschließender Vergleich zwischen Vertretern des Freistaats Sachsen und des Hauses Wettin geschlossen, der den Verbleib fast aller fraglichen Stücke im Eigentum des Freistaats sicherte. ${ }^{19}$

19 Vgl. Gemeinsame Medieninformation des Sächsischen Staatsministeriums für Wissenschaft und Kunst, des Sächsischen Staatsministeriums der Finanzen und des Hauses Wettin A. L. vom 17. Juli 2014. https://www.medienservice.sachsen.de/medien/news/193289 (4.1.2018). 